\title{
INFLUENCE DES VARIATIONS D'OXYGENE DISSOUS SUR LES INVERTÉBRẼS BENTHIQUES D'UN RUISSEAU DES PYRÉNÉES CENTRALES
}

\author{
par P. Lavandier ${ }^{1}$ et J. Capblance ${ }^{1}$.
}

\begin{abstract}
Des expériences de désoxygénation partielle de l'eau d'un ruisseau montrent qu'il existe un seuil de concentration en $\mathrm{O}_{2}$ dissous (ici proche de $4 \mathrm{ppm}$ ) au-dessous duquel la dérive des invertébrés benthiques s'accroit de façon significative. L'importance de cette dérive est alors variable selon les espèces considérées, l'intensité et la durée de la désoxygénation. Les variations quotidiennes des teneurs en $\mathrm{O}_{2}$ dissous que présentent naturellement les eaux courantes, ne paraissent entrainer aucune réaction de la faune si elles se situent au-dessus de ce seuil.
\end{abstract}

\section{Effect of variations in dissolved oxygen on the benthic invertebrates} of a stream in the central Pyrenees.

Some experiments with the partial deoxygenation of the water of a stream showed that there is a threshold for dissolved oxygen (close to $4 \mathrm{ppm}$ ) below which the drift of benthic invertebrates significantly increases. The magnitude of the drift varies between species, and with both the intensity and duration of the deoxygenation. The daily variations in dissolved oxygen content occurring naturally in running water do not appear to affect the fauna if the oxygen content is higher than the threshold given above.

\section{INTRODUCTION}

Dans les conditions naturelles, la teneur en oxygène dissous des eaux courantes présente des variations quotidiennes plus ou moins amples suivant la turbulence, les fluctuations thermiques et le peuplement végétal des cours d'eau. En outre, l'abaissement des concentrations en oxygène résultant de pollutions organiques est un phénomène de plus en plus fréquent. Au cours de l'établissement du bilan en oxygène d'un petit ruisseau (Capblancq et Lavandier en prép.) nous avons utilisé la méthode au sulfite (Gameson et al. 1955, Edwards et al. 1961) pour calculer la diffusion. Ces expériences nous ont permis d'apprécier l'effet de désoxygénations brèves et plus ou moins importantes sur la faune benthique au travers de la dérive des organismes.

1. Laboratoire d'Hydrobiologie, Université Paul-Sabatier, 118, route de Narbonne, 31077 Toulouse Cedex. 


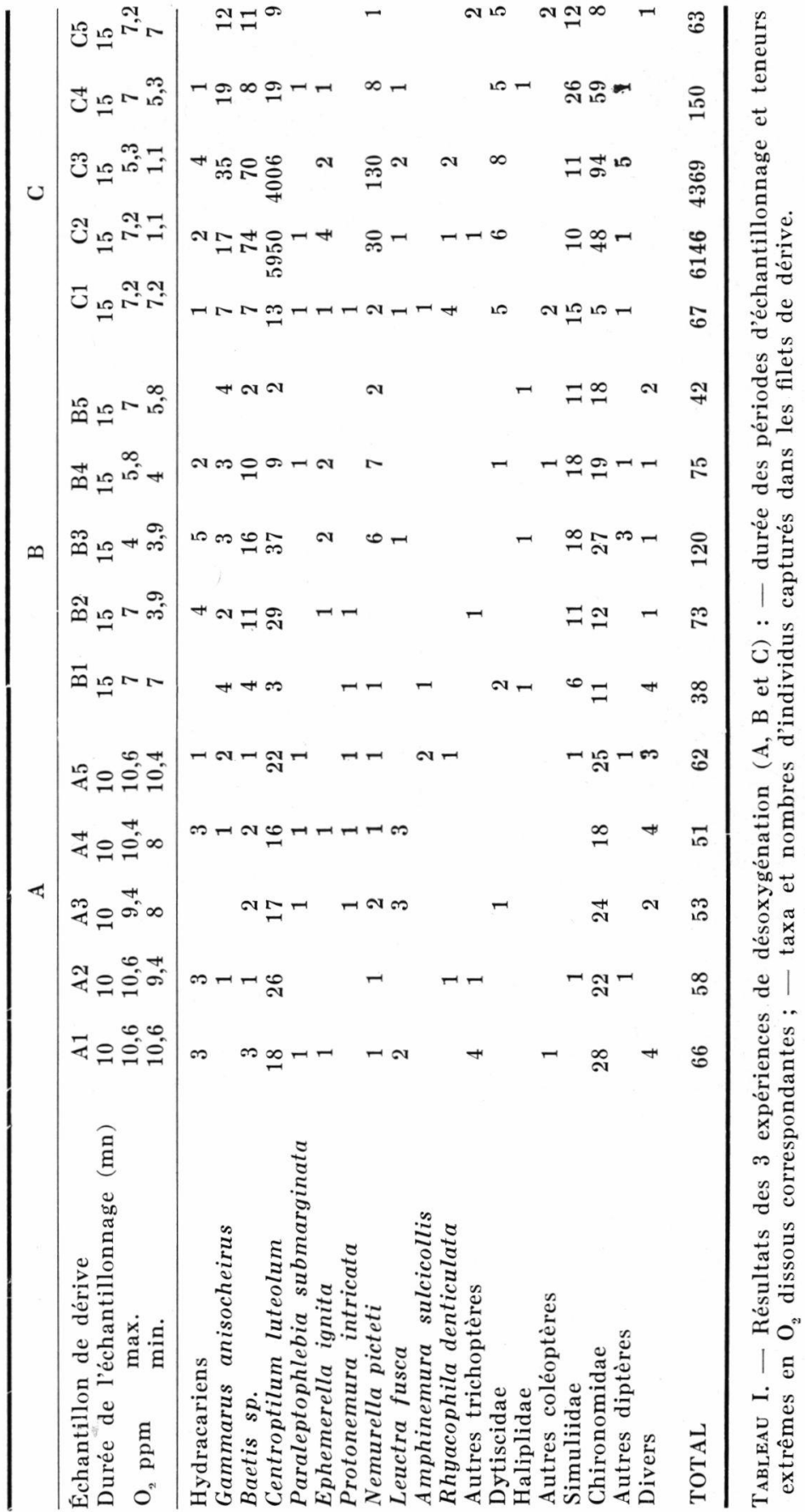




\section{MÉTHODES}

L'addition dans une eau d'unc solution de sulfite de sodium et d'un catalyseur (sel de cobalt) détermine après un temps de réaction une diminution de la teneur en oxygène dissous. Dans un ruisseau, elle provoque une vague de désoxygénation dont l'étendue dépend de la durée du déversement el dont l'importance est fonction de la quantité de sulfite ajoutée et de la distance au point d'injection.

Nous avons réalisé nos mesures en mars et avril dans un petit ruisseau des Pyrénées centrales dont les caractérisliques physicochimiques et biologiques ont été décrites précédemment (Lavandier et Dumas 1972 a et b). Une solution de sulfite de sodium et de nitrate de cobalt était déversée par un appareil à débit constant, placé en amont d'une section dont les caractéristiques morphodynamiques sont les suivantes :

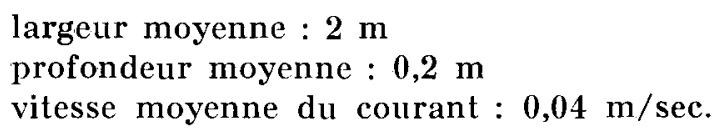

L'échantillonnage de la dérive et les dosages d'oxygène dissous étaient effectués en aval du point de déversement, dans une zone correspondant à la plus forte désoxygénation. Un filet barrait intégralement le ruisseau 15 mètres en amont du point d'échantillonnage, afin de ne récolter que la faune en provenance de cette zone. Un filet de contrôle était placé 40 mètres en aval.

Trois expériences $(A, B, C)$ ont été réalisées avec des quantités croissantes de sulfite et une quantité constante de nitrate de cobalt qui déterminait dans le ruisseau une teneur voisine de $0,5 \mathrm{mg} / \mathrm{l}$. Cinq échantillons de dérive ont été recueillis lors de chaque expérience, le premier et le dernier correspondant à des conditions d'oxygénation normales.

La température de l'eau était de $10^{\circ} \mathrm{C}$.

\section{RESULTATS}

Les résultats de dérive et les conditions d'oxygénation sont donnés dans le tableau I et la figure 1.

Un test de $\chi^{2}$ de conformité avec une distribution de Poisson a été appliqué aux résultats de chaque série d'échantillons (voir Elliott 1970). Cette conformité n'est pas réalisée pour les expériences $\mathrm{B}$ et $\mathrm{C}$, que l'on considère le nombre total d'individus capturés ou l'effectif des espèces dominantes. Par contre, les résultats correspondants de l'expérience $A$, ainsi que ceux relatifs aux échantillons B1 et B5 d'une part, C1 et C5 d'autre part sont conformes 


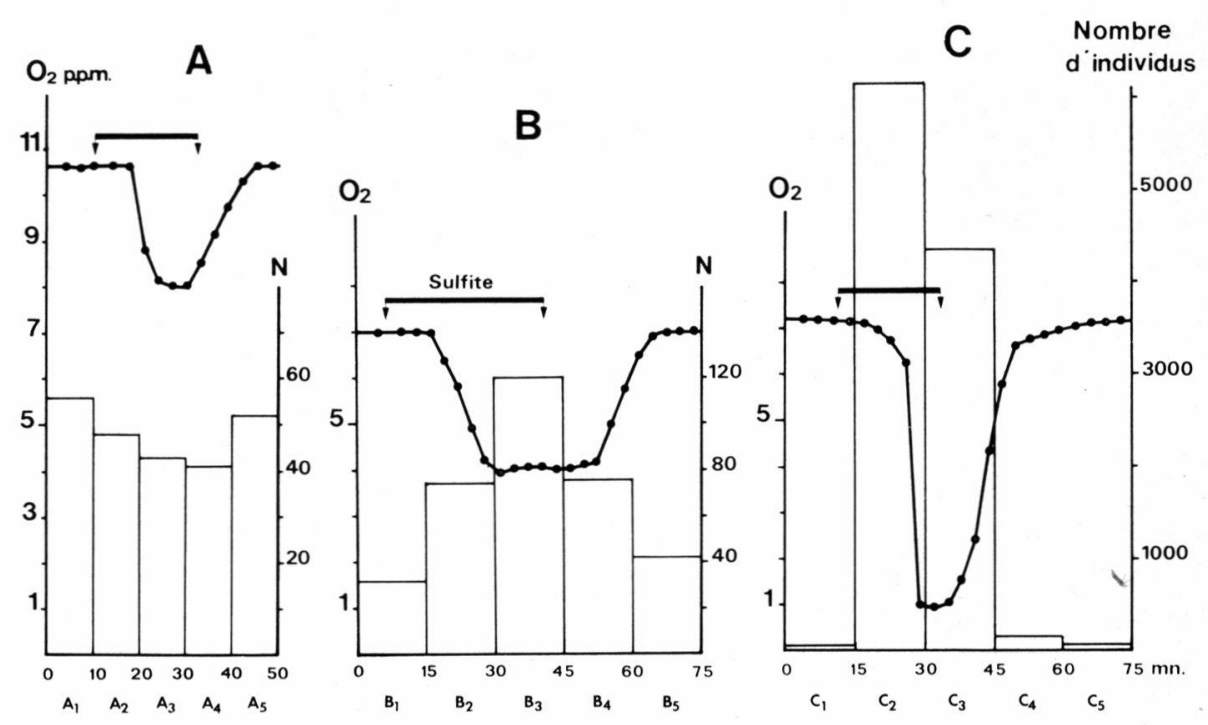

Fig. 1. - Évolution des teneurs en $\mathrm{O}_{2}$ dissous (courbes) et du nombre d'invertébrés capturés dans les filets de dérive (histogrammes), lors des 3 expériences ( $A$, B et $C$ ) de désoxygénation au sulfite.

à une telle distribution. Cette analyse montre donc 1) qu'en dehors de toute influence extérieure la dérive est restée constante pendant la durée de chaque expérience; 2) que le sel de cobalt utilisé comme catalyseur n'a aucune influence sur la dérive.

Pour les expériences $\mathrm{B}$ et $\mathrm{C}$, un test de comparaison de deux séries de Poisson (voir Décamps et Elliott 1972) a permis de comparer l'abondance des organismes capturés pendant et en dehors de la phase de désoxygénation. Dans les deux cas, l'augmentation du nombre total d'individus et de l'effectif des espèces dominantes (Baetis sp., Centroptilum luteolum, Nemurella picteti) est hautement significative. L’intensité de la dérive est fonction des teneurs minimales en oxygène dissous : la dérive est multipliée par 3 ou 4 dans l'expérience B, par plus de 100 dans l'expérience C.

Il existe quelques différences selon les taxa. L'augmentation du nombre de Chironomides est significative lorsque la teneur en $\mathrm{O}_{2}$ descend à 4 ppm, hautement significative lorsqu'elle descend à $1 \mathrm{ppm}$ (exp. C). La dérive de Gammarus anisocheirus n'augmente de façon significative que lors de l'expérience C. Celle des Simulies, difficilement explicable, s'accroît significativement dans l'expérience B (4 ppm) alors qu'elle n'est pas modifiée au cours de l'expérience $\mathrm{C}$. 


\section{DISCUSSION}

L'effet d'imporlantes variations des teneurs en oxygène dissous sur la faune et les différences spécifiques que nous avons noté ne sont pas surprenantes. La comparaison des trois expériences permet cependant de préciser ces observations.

- La faible désoxygénation (10,6 à $8 \mathrm{ppm})$ provoquée lors de l'expérience A ne détermine aucune réaction apparente chez les invertébrés benthiques. Notons qu'elle es! comparable aux fluctuations quotidiennes de concentration en $\mathrm{O}_{2}$ dissous couramment observées dans les ruisseaux ; il ne semble donc pas que, dans les conditions naturelles, la diminution des teneurs en $O_{2}$ consécutives à l'arrêt de l'activité pholosynthétique des végétaux en fin d'aprèsmidi joue un rôle sur le rythme nycthéméral de la dérive.

-. La teneur de 4 ppm atteinte dans l'expérience B est certainement proche du «seuil de décrochage» pour la faune benthique en place. La résistance des invertébrés à ces «conditions limites » varie selon les espèces mais il est remarquable de constater que, pour tous les taxa, le nombre maximum d'organismes en dérive correspond à la seule période d'échantillonnage où l'eau a été maintenue à $4 \mathrm{ppm}$.

- Pour des teneurs inférieures (expérience $C$ ) les invertébrés sont très rapidement entraînés par le courant. La durée de résistance est ici très courte puisque le filet $\mathrm{C} 2$ qui a recueilli le nombre d'organismes le plus élevé n'a filtré de l'eau fortement désoxygénée que pendant 2 minutes environ. Dans cette dernière expérience, il faut également signaler que la faune capturée a repris très vite une activité apparemment normale dès le relour aux conditions d'oxygénation initiales. Le filet de contrôle placé 40 mètres en aval ne présentait par ailleurs qu'une faible augmentation de la dérive.

Ces notions classiques d'intensité et de durée d'un stimulus appliquées aux teneurs en $\mathrm{O}_{2}$ dissous doivent être soulignées à une époque où l'altération des conditions d'oxygénation est un phénomène de plus en plus fréquent. Une chute importante des teneurs en oxygène entraîne très rapidement un dépeuplement du fond, même si la faune benthique n'est pas tuée. Les teneurs intermédiaires provoqent une dérive plus faible que dans le cas précédent, mais leur effet est d'autant plus sensible que leur action es! prolongée. Il est ainsi possible que les faibles diminutions des concentrations en $\mathrm{O}_{\mathrm{a}}$ dissous, aient à longue échéance une influence de même nature, influence que nous n'avons pu mettre en évidence étant donné la courte durée d'expérimentation. 


\section{TRAVAUX CITÉS}

Capblance (J.) et Lavandier (P.). - Production primaire et bilan en oxygène dissous dans un ruisseau des Pyrénées centrales (en préparation).

Décamps (H.) et Elliott (J. M.). 1972. - Influence de la mesure chimique du débit sur les invertébrés d'un ruisseau de montagne. Annls Limnol., 8 (3) : 217-222.

Edwards (R. W.), Owens (M.) et Gibbs (J. W.). 1961. - Estimates of surface aeration in two streams. J. Inst. Wat. Engrs. 15 : 395-405.

Elliott (J. M.). 1970. - Methods of sampling invertebrate drift in running water. Annls Limnol., 6 (2) : 133-159.

Gameson (A. L.H.), Truesdale (G. A.) et Downing (A. L.). 1955. Reaeration studies in a lakeland beck. J. Inst. Wat. Engrs. 9 : $57-94$.

Lavandier (P.) et Dumas (J.). 1971. - Microrépartition de quelques espèces d'invertébrés benthiques dans des ruisseaux des Pyrénées centrales. Annls. Limnol., 7 (1) : 7-23.

Lavandier (P.) et Dumas (J.). 1971. - Cycles de développement de quelques invertébrés benthiques dans des ruisseaux des Pyrénées centrales. Annls. Limnol., 7 (2) : 157-172. 